\title{
Tratamento farmacológico e não farmacológico da disfunção temporomandibular: uma revisão da literatura
}

Pharmacological and non-pharmacological treatment of temporomandibular disorder: a literature review Tratamiento farmacológico y no farmacológico de la disfunción temporomandibular: una revisión de la literatura

Ozanna Soares Medeiros de ARAUJO' ${ }^{1}$

José Henrique de Araújo $\mathbf{C R U Z}^{2}$

Abrahão Alves de OLIVEIRA FILHO ${ }^{3}$

Maria Angélica Satyro Gomes ALVES ${ }^{3}$

${ }^{1}$ Acadêmico(a) do Curso de Graduação em Odontologia. Centro de Saúde e Tecnologia Rural, Universidade Federal de Campina Grande, UFCG 58708-110 Patos - PB, Brasil

${ }^{2}$ Cirurgião-Dentista e Pós-Graduando em Ortodontia. Centro de Saúde e Tecnologia Rural, Universidade Federal de Campina Grande, UFCG 58708-110 Patos - PB, Brasil

${ }^{3}$ Professor(a) Doutor(a) do Curso de Graduação em Odontologia. Centro de Saúde e Tecnologia Rural, Universidade Federal de Campina Grande UFCG 58708-110 Patos - PB, Brasil

\section{Resumo}

Introdução: A disfunção temporomandibular tem sido reconhecida como uma das causas das dores orofaciais, nesta condição existe o envolvimento da articulação temporomandibular como também os músculos da mastigação. Esta disfunção têm sido identificado por alguns sintomas e sinais característicos como, pontos de gatilho musculares, comprometimento da dimensão vertical de oclusão, modificações na oclusão dental, luxação e a subluxação da mandíbula. Objetivo: Este trabalho objetiva a identificação e a análise dos tratamentos farmacológicos (anti-inflamatórios não esteroidais; anti-inflamatórios esteroidais; antidepressivos tricíclicos; relaxantes musculares e também os anestésicos locais) e não farmacológicos, estes incluídos no tratamento alternativos utilizados na disfunção temporomandibular (Acupuntura, Toxina botulínica, Estimulação nervosa elétrica transcutânea, e Técnicas de massoterapia. Material e métodos. Metodologia: Foram escolhidos 41 artigos presentes em plataformas cientificas, como o Periódico Capes, PubMed, Scielo e Google Acadêmico, no período de publicação entre 2006 e 2019. Conclusão: Por fim, nota-se que a disfunção temporomandibular é uma condição que acomete muito a população e que sua terapia pode ser realizada de diversas formas, desde com a terapia medicamentosa como também a realização de tratamentos alternativos.

Descritores: Transtornos da Articulação Temporomandibular; Dor Facial; Terapêutica.

\section{Abstract}

Introduction: Temporomandibular dysfunction has been recognized as one of the causes of orofacial pain. In this condition we have involvement of the temporomandibular joint as well as the chewing muscles. This dysfunction has been identified by some characteristic symptoms and signs such as muscle trigger points, impairment of the vertical occlusion dimension, changes in dental occlusion, dislocation and jaw subluxation. Objective: This study aims to identify and analyze pharmacological treatments (non-steroidal anti-inflammatory drugs; steroidal anti-inflammatory drugs; tricyclic antidepressants; muscle relaxants as well as local anesthetics) and non-pharmacological treatments, which are included in the alternative treatments used in temporomandibular dysfunction (Acupuncture, Botulinum Toxin, Transcutaneous Electrical Nerve Stimulation, and Massotherapy Techniques Material and Methods Methodology: 41 articles from scientific journals such as Capes, PubMed, Scielo and Google Scholar were chosen from 2006 to 2019. Conclusion: Finally, it is noted that temporomandibular dysfunction is a condition that greatly affects the population and that its therapy can be performed in various ways, from drug therapy to alternative treatments.

Descriptors: Temporomandibular Joint Disorders; Facial Pain; Therapeutics.

\section{Resumen}

Introducción: la disfunción temporomandibular ha sido reconocida como una de las causas del dolor orofacial, en esta afección tenemos afectación de la articulación temporomandibular y de los músculos masticatorios. Esta disfunción ha sido identificada por algunos síntomas y signos característicos, como puntos gatillo musculares, deterioro de la dimensión de oclusión vertical, cambios en la oclusión dental, dislocación y subluxación de la mandíbula. Objetivo: Este estudio tiene como objetivo identificar y analizar tratamientos farmacológicos (medicamentos antiinflamatorios no esteroideos; medicamentos antiinflamatorios esteroideos; antidepresivos tricíclicos; relajantes musculares, así como anestésicos locales) y tratamientos no farmacológicos, que se incluyen en los tratamientos alternativos utilizados en la disfunción temporomandibular ( Acupuntura, toxina botulínica, estimulación nerviosa eléctrica transcutánea y técnicas de masoterapia Material y métodos Metodología: se eligieron 41 artículos de revistas científicas como Capes, PubMed, Scielo y Google Scholar de 2006 a 2019. Conclusión: Finalmente, se observa que la disfunción temporomandibular es una condición que afecta en gran medida a la población y que su terapia se puede realizar de varias maneras, desde la farmacoterapia hasta los tratamientos alternativos.

Descriptores::Trastornos de la Articulación Temporomandibular; Dolor Facial; Terapéutica.

INTRODUÇÃO

$$
\text { A disfunção temporomandibular (DTM) é }
$$
uma das causas mais recorrentes da dor orofacial, envolvendo os músculos da mastigação e a articulação temporomandibular (ATM). Como fatores desencadeadores dessa disfunção destacam-se os traumatismos locais, as maloclusões e também os aspectos psicológicos. Os sintomas iniciais percebidos da DTM são barulhos condilares, pontos de gatilho musculares, comprometimento da dimensão vertical de oclusão, ausência e movimentações de dentes, modificações na oclusão dental, contenção e os desvios na abertura bucal, manobras mandibulares sem coordenação, luxação e a subluxação da mandibula ${ }^{1}$.

A DTM é classificada de três formas Dor miofascial, distúrbios internos da ATM, e doenças degenerativas da ATM. A dor miofascial caracteriza-se como uma dor nos músculos mastigatórios que estão intimamente ligados à mandíbula; os distúrbios internos da ATM estão relacionados com o disco articular; e as doenças degenerativas da articulação temporomandibular relacionam-se com algumas 
doenças que tendem a atingir o indivíduo na terceira idade, como osteoartrite e artrite reumatóide ${ }^{1}$.

Destaca-se a recorrência de DTMs em indivíduos de 20 a 45 anos, tendo como origem muscular sua principal causa em pessoas de até 40 anos. Acima desta faixa etária evidenciase que seu principal fator se torna a degeneração articular $^{2}$. Em relação à população, destaca-se que $37,5 \%$ possua um sintoma de DTM e que entre os universitários esse índice se eleva para valores entre $41,3 \%$ e $68.6 \%{ }^{3}$.

$\mathrm{Na}$ disfunção temporomandibular existem diferentes classes de medicamentos que atuam na melhora desta desordem, como os analgésicos; anti-inflamatórios; relaxantes musculares e também antidepressivos, visto que estes fármacos colaboram em proporcionar um bem-estar e a reabilitação do paciente, ainda assim, alguns estudiosos como o Okeson ${ }^{4}$ afirmam que nenhuma substância se provou capaz de ser eficiente isoladamente contra todos os sintomas da DTM.

Segundo uma pesquisa realizada por Bouloux ${ }^{5}$, observou-se a relação de opióides com a DTM, destacando que o seu uso a longo prazo em alguns casos de pacientes com dor crônica não oncológica, pode melhorar a função da articulação e estabelecer uma melhor qualidade de vida.

Ainda de acordo com Okeson ${ }^{4}$, foram definidos alguns tipos de medicamentos para sintomas específicos da DTM. O autor afirma que os analgésicos, ansiolíticos e também os corticoides são indicados para tratar a dor aguda. Já em relação a dor crônica, evidenciase a atuação de antidepressivos tricíclicos.

Diante do exposto, objetivou-se pesquisar e organizar de forma didática todas as classificações medicamentosas e não medicamentosas encontradas nas plataformas acadêmicas que tenham o fim de tratar a disfunção temporomandibular.

MATERIAL E MÉTODO

Segundo $\mathrm{Gil}^{6}$, podemos classificar este estudo como uma pesquisa bibliográfica, pois ela foi elaborada com base em material já produzido. Para a realização desta revisão bibliográfica narrativa, foram eleitos artigos presentes em plataformas cientificas, como o Periódico Capes, PubMed, Scielo e Google Acadêmico. Nestas bases de dados utilizaramse palavras chaves, como: "disfunção temporomandibular (temporomandibular dysfunction)"; "analgesia da DTM (analgesia of the TMD)"; e também "farmacolgia na DTM (pharmacology in the TMD)". Após as buscas, foram selecionados 41 artigos no período de publicação entre 2006 e 2019 que estão relacionados ao tema estudado, sendo eles na língua portuguesa e inglesa. Esta seleção ocorreu no período de outubro de 2018 a janeiro de 2019.

Em seguida para melhor compreensão, foi feita uma divisão em tópicos dos fármacos no tratamento da DTM. Nessa divisão dos fármacos utilizados na DTM, foram elucidadas características específicas destes medicamentos e também a sua forma de atuação no corpo humano, estando entre eles os: anti-inflamatórios não esteroidais; antiinflamatórios esteroidais; antidepressivos tricíclicos; relaxantes musculares e também os anestésicos locais. Além da terapia medicamentosa também foram elucidados os tratamentos alternativos utilizados contra a disfunção temporomandibular.

RESULTADOS E DISCUSSÃO

- Terapia Medicamentosa

- Medicamentos

Os medicamentos são produtos elaborados a partir de fármacos, que apresentam fins comerciais. Eles são desenvolvidos com o objetivo de proporcionar ao paciente melhor qualidade nos serviços de saúde, através da prevenção e monitoramento dos possíveis danos à saúde e também da promoção e implementação de ações que estejam relacionadas com a segurança do indivíduo ${ }^{7}$. Segundo a ANVISA $^{8}$, os medicamentos são produzidos com finalidade de diagnosticar, prevenir, curar doenças ou aliviar a sintomatologia do enfermo, sendo eles elaborados com rígido controle técnico para atender às especificações determinadas por esse órgão.

E comum haver confusão nos conceitos de "remédio" e "medicamento", sendo eles utilizados como sinônimos um do outro. Como foi esclarecido, o medicamento é aquele que possui uma garantia de ação, que foi percebida no controle técnico. Já o remédio, ele possui um conceito mais amplo, pois pode significar qualquer ação realizada no indivíduo ou praticada por ele, mesmo que o faça se sentir melhor, como por exemplo, uma massagem, um ritual religioso, chás e inclusive $o$ uso de medicamentos ${ }^{9}$.

Conforme afirmam Sharma et $\mathrm{al}^{10}$., ao tomar a medicação errada ou forma incorreta, muitos danos são gerados ao paciente e esse erro pode ser acometido por diversos fatores, como: falta de atenção e concentração, falha na 
comunicação entre o paciente e o profissional de saúde, ausência de informações sobre o medicamento e também sobre 0 não conhecimento do histórico de doenças do paciente. Complementando o que Sharma et al. ${ }^{10}$ colocam, Teixera e Cassini ${ }^{11}$ explanam que os erros podem advir de uma prescrição, da distribuição, administração de medicamentos e inclusive o monitoramento do paciente.

De acordo com a ANVISA ${ }^{8}$ acerca do valor monetário desses medicamentos, a Câmara de Regulação do Mercado de Medicamentos (CMED) é o orgão que busca evitar que os preços dos medicamentos estejam em demasia e também coibir os reajustes não autorizados. Apesar desse controle, segundo o $\mathrm{IBGE}^{12}$, no Brasil a despesa com medicamentações ainda ocupam cerca de $46,6 \%$ de toda a despesa familiar.

\section{- Anti-inflamatórios não esteroidais (AINEs)}

Segundo Lynch e Watson ${ }^{13}$ os antiinflamatórios não esteroidais (AINEs) são os medicamentos mais receitados pelos cirurgiõesdentistas devido o seu poder analgésico e antiinflamatório. O seu mecanismo funciona pela inibição de uma ou ambas isoformas da enzima ciclo-oxigenase (COX), e o efeito analgésico é obtido devido à redução da síntese de mediadores inflamatórios do tipo prostaglandinas. Contudo, este fármaco apresenta efeitos colaterais, pois a inibição da isoforma da COX-1, COX-2, na maioria das vezes não é seletiva.

Para Hilário et al. ${ }^{14}$ as duas isoformas encontradas da COX são denominadas de COX-1 e COX-2. A enzima cicloxigenase-1 está presente em vários tecidos e por isso é designada de constitutiva, ela têm a função de promover a homeostasia. Em relação a COX-2, a sua existência não é constante no corpo, pois ela só está presente quando ocorre uma inflamação, já que essa enzima é induzida pelo processo inflamatório. De acordo com Carvalho $^{15}$ os efeitos anti-inflamatórios e analgésicos ocorrem devido a inibição da enzima COX-2 e os efeitos não desejados ocorrem principalmente devido a inibição da enzima COX-1.

Okeson ${ }^{4}$ determinou que quando os AINEs são administrados na dose adequada, eles podem se tornar úteis no tratamento das desordens inflamatórias da ATM. Por outro lado, Batlouni $^{16}$ afirma que a ingestão diária deste fármaco pode gerar uma tolerância e contribuir com o aparecimento de efeitos adversos, como por exemplo, efeitos cardiovasculares e problemas gastrointestinal.

Dym e Israel $^{17}$ listam os AINEs mais utilizados na odontologia pra o tratamento da DTM são: Diclofenaco; Diflunisal; Etodolaco; Fenoprofeno; Ibuprofeno; Cetorolaco; Naproxeno;Piroxicam e Sulindaco. Lynch e Watson ${ }^{13}$ afirmam que entre os antiinflamatórios não esteroidais existe um subgrupo denominado COXIBEs, o qual possui somente agentes seletivos inibidores da enzima COX2, estes medicamentos foram projetados com o objetivo de diminuir os efeitos colaterais gastrointestinal, comumente ligados aos AINEs já conhecidos. Complementando o que Lynch e Watson ${ }^{13}$ afirmaram, Yagiela et al. ${ }^{18}$ informam que celecoxib e rofecoxib são algumas das formas que os COXIBs se apresentam no mercado.

- Anti-inflamatórios esteroidais (corticosteróides) Segundo Yagiela et al. $^{18}$ os corticosteróides ou corticóides são subdivididos em dois grupamentos, os mineralocorticóides e glicocorticóides, destacando este último como os únicos que possuem efeitos antiinflamatórios. Outrossim, nesta mesma linha de raciocínio, Kazung ${ }^{19}$ elenca que a ação desta classe de corticosteróides atua na inibição da enzima fosfolipase A2, no qual ativa a produção de ácido araquidônico, este ácido tem a responsabilidade de sintetizar as prostaglandinas (PGs), que causam a sensação de dor, além de atuar nesta enzima, eles também agem inibindo indiretamente a COX.

De acordo com Yagiela et al. $^{18}$ essa dupla atuação garante a diminuição da quimiotaxia celular e também a inibição da resposta vascular e inflamatória às PGs. Ele ainda informa que dentre os glicocorticóides mais indicados para a terapia da DTM, destacase a hidrocortisona, prednisolona, dexametasona e betametasoma.

Os efeitos colaterais gerados por essa divisão de anti-inflamatórios esteroidais são de intolerância à glicose; úlceras pépticas; cataratas e também o aumento do risco de contágio com infecções oportunistas e dentre outros. Dentre os glicocorticóides evidencia-se a dexametasona e betametasona como fármacos nesta categoria que apresentam efeitos colaterais em menor nível ${ }^{20}$.

- Antidepressivos tricíclicos (ADT)

Conforme afirmam Garrote et al. $^{21}$, atualmente os antidepressivos têm sido usados não somente em casos de depressão mas também em tratamentos de dores crônicas, como por exemplo a amitriptilina . Os antidepressivos tricíclicos mais utilizados na odontologia para tratar a disfunção temporomandibular são Amitriptilina; Nortriptilina e Imipramina. Melo ${ }^{22}$ complementa ainda 
dizendo que a prescrição destes medicamentos tem sido feita para dores crônicas e também para pacientes que apresentam bruxismo e distúrbio do sono.

Segundo Medawar e Matheus ${ }^{23}$, os mecanismos de ação dos ADTs baseiam-se no bloqueio da recaptação da noradreanalina e da serotonina na fenda pós-sináptica. A ação sobre estas monoaminas (noradrenalina e serotonina) possibilita que as mesmas possam interagir com seus receptores específicos presentes nos Inter neurônios inibitórios que se localizam no corno dorsal da medula, desse modo ocorrerá um aumento de sinapses e, por conseguinte, a amplificação do limiar de ativação dos neurônios secundários situados na via nociceptiva. Assim sendo, a condução da dor que se direcionada ao tálamo fica prejudicada e a resposta da dor se torna em menor grau.

Em contrapartida, Goodman e Gilman ${ }^{24}$ afirmam que as desvantagem do uso desses fármacos está na sua interação com outros receptores, como os adrenérgicos, muscarínicos e histamínicos, essa interação acarreta em vários efeitos colaterais. Como por exemplo, os efeitos cardiovasculares (hipotensão ortostática, taquicardia sinusal e prolongamento variável da condução cardíaca com riscos de desenvolvimento de arritmias); efeitos antimuscarínicos (i.e. xerostomia, desconforto epigástrico, constipação, retenção urinária, taquicardia, tontura, palpitações e borramento visual) e também os efeitos anti-histamínicos (aumento do apetite e sedação). Entre estes medicamentos os que apresentam menores efeitos colaterais são a amitriptilina e a nortriptilina

\section{- Relaxantes musculares}

Cascaes e Oliveira ${ }^{25}$ concordam que os relaxantes musculares constituem uma classe de ampla atuação, sendo empregados em condições musculoesqueléticas periféricas, fibromialgia, espasticidade e também dores neuropáticas, como a neuralgia trigeminal. Segundo $\mathrm{Melo}^{22}$, a utilização desses farmacos induz a um relaxamento central dos músculos devido à supressão de uma porção dos impulsos nervosos para os músculos estriados.

Rang e Ritter ${ }^{26}$ classificam estes medicamentos se subdividem em relaxantes musculares de ação periférica e de ação central. Os de ação periférica também conhecidos como bloqueadores neuromusculares agem interferindo na transmissão de impulsos nervosos que ocorrem na junção neuromuscular. Os relaxantes musculares de ação central atuam na diminuição da corrente tônica que ocorre dos impulsos nervosos do
SNC aos músculos esqueléticos, essa supressão parcial dos impulsos afetam a atividade neural que está associada aos reflexos de estiramento muscular, diminuindo o tônus.

\section{- Anestésicos locais}

Andrade et al. $^{1}$ asseguram que os anestésicos locais são rotineiramente utilizados pelos cirurgiões dentistas para o tratamento das dores resultantes da DTM. Estes fármacos têm a função de suprimir a condução dos estímulos nervosos do sistema nervoso periférico ou central de forma reversível, garantindo que uma área específica esteja dessensibilizada. $\mathrm{O}$ mecanismo de ação desta droga se dá por estes atravessarem a membrana do axônio e adentrando na célula nervosa, dentro desta célula o anestésico local irá se ligar aos receptores específicos presentes nos canais de sódio e desse modo têm se o impedimento da passagem de sódio para o interior da célula. Consequentemente ocorre 0 bloqueio da condução nervosa e em decorrrência deste bloqueio ocorre a diminuição da sensação de dor.

Segundo Okeson ${ }^{4}$, uma injeção de anestésico em um ponto de gatilho miofascial (PGM) tem a capacidade de diminuir a sensação de dor do paciente. Os pontos de gatilhos miofascial são áreas determinadas do músculo que possui a dor de mesmo nome. De acordo com 0 autor Veering ${ }^{27}$ os anestésicos mais utilizados na odontologia são "lidocaína, prilocaína, mepivacaína e a bupivacaína. Incluem-se ainda a articaína, a ropivacaína e a levobupivacaína". A lidocaína é usualmente utilizada nos PGM.

Andrade et al. ${ }^{1}$ discorrem que os efeitos adversos resultantes do uso deste medicamento são praticamente inexistentes se forem aplicados na dosagem correta. No entanto, se ocorrer uma sobredosagem ou o uso de técnicas anestésicas incorretas, os efeitos colaterais podem se tornar prejudiciais, podendo levar á neurotoxidade ou cardiotixicidade. Os efeitos colaterais encontrados podem ser reações alérgicas, toxidade sistêmica devido a depressão do sistema nervoso central (SNC) e em relação à injeção intravascular acidental, têm se o aumento de níveis plasmáticos de anestésicos no sangue. Nesta mesma linha de raciocínio acerca dos efeitos adversos, Muri et al. $^{28}$ ainda colocam que os anestésicos locais podem produzir agitação, nervosismo e tremor.

- Tratamento alternativos utilizados na DTM

- Acupuntura

Zotelli et al. ${ }^{29}$ declaram que na DTM o mecanismo de ação da acupuntura se baseia na 
estimulação de determinados pontos em que são utilizados agulhas muito finas na punção, essa prática busca a estimulação nas terminações nervosas a liberar neurotransmissores que possuem características benéficas quanto a manutenção do bem estar físico, como por exemplo, a liberação de endorfinas e cortisol, que possuem propriedades analgésicas. A estimulação desses pontos também promove a alteração da dinâmica presente na circulação e também proporciona a redução da dor e da inflamação.

É importante ressaltar que essa Medicina Tradicional Chinesa (MTC) não objetiva agir sobre um problema específico em determinado local no corpo, mas sim sobre o sistema nervoso, levando esse organismo ao equilíbrio.

Em uma pesquisa realizada por Borien et al. $^{30}$, houve a distribuição de 40 mulheres em dois grupos, um grupo realizava o tratamento com acupuntura duas vezes na semana e o outro grupo só realizou esse tratamento após o mesmo ser feito no primeiro grupo, servindo esse segundo como grupo de controle. Nesse estudo Borien relatou que houve a melhora significativa da dor, e essa analgesia pode ser explicada devido essa prática agir no eixo hipotálamo-pituitárioadrenal, no qual tem a ativação dos centros corticais assim como a liberação de substancias anti-inflamatórias.

Florian et al. ${ }^{31}$ reiteram que essa técnica vem sendo muito utilizada pela odontologia devido seus aspectos benéficos já comprovados, além disso, o tratamento por meio da medicina tradicional chinesa pode ser configurado como não invasivo e reversível e também ser uma terapia segura, individualizada e ainda assim agir tanto localmente quanto no fator de estresse emocional.

\section{- Toxina botulínica}

Oliveira $^{32}$ explica que a Neurotoxina botulínica é concebida pela bactéria Clostridium botulinum, um organismo anaeróbico. Em 1920 foi descoberta sete formas dessa toxina $(A, B$, $C, D, E, F$ e G) sendo a toxina A mais potente e com uma maior duração. Segundo Gonçalves ${ }^{33}$, essa toxina vem comumente sendo utilizada na odontologia para tratar casos de DTM, dor orofacial, bruxismo, sorriso gengival, queilite angular, sorriso assimétrico, hipertrofia de masseter, pós-operatório de cirurgias periodontais e de implantes.

Bicalho et al. $^{34}$ certificam que as aplicações da toxina botulínica promovem um conforto e também um alívio aos pacientes que relatam dores oriundas das DTM's. A sua administração ocorre pela via subcutânea, tendo o prazo de funcionamento dentre 3 a 6 meses, ela se configura como uma técnica pouco invasiva e que exibe aspectos positivos no tratamento. Na odontologia o seu uso só foi permitido a partir do ano de 2011.

Dall' Antonia et al. ${ }^{35}$ informam que a ação dessa substância foi desvendada na década de 50, e descobriu-se então que ela agia bloqueando a liberação de acetilcolina nas terminações nervosas motoras e que ao adentrar no organismo a toxina buscava a junção neuromuscular e nessa região se ligava ao receptor, bloquendo assim a transmissão do impulso nervoso. Apesar de eficaz, Dall' Antonia et al. $^{35}$ ressaltam que dentre as restrições de seu uso estão as mulheres que estão grávidas ou em período de lactação, pessoas que possuem inflamação no local de injeção proposto, indivíduos que realizam terapia com anticoagulantes ou então que tomam medicamentos que possuam alguma interferência na transmissão neuromuscular.

- Estimulação nervosa elétrica transcutânea (TENS)

De acordo com Almeida ${ }^{36}$, a estimulação nervosa elétrica transcutênea (TENS) é um tratatamento que realiza a estimulação elétrica por intermédio da pele para obter o controle da dor. Ela possui várias características positivas, como por exemplo: não apresentar efeitos secundários; não ser invasivo, além de apresentar facilidade em seu uso. A estimulação elétrica é classificada em dois níveis, nível sensorial e nível motor.

Ainda, conforme Almeida ${ }^{36}$ explica, no nível sensorial a voltagem se eleva até provocar uma parestesia ou sensação de toque. Já em relação ao nível motor, a voltagem é aumentada até chegar a uma contração motora. $O$ mecanismo de ação dessa técnica pode ser explicado por duas teorias, a liberação de endorfina endógena induzida por estimulação e o outro pelo controle do portão para a dor.

Segundo Grossmann et al. ${ }^{37}$, a teoria do controle de portão foi publicada em 1965 e afirmava que a ativação das fibras nervosas mielinizadas aferentes que possuíam largo diâmetro iria atuar sobre uma substância gelatinosa presente no corno dorsal, e isso provocaria a inibição do impulso transmitido pelas fibras nociceptivas não mielinizadas que possuíam pequeno diâmetro, inibindo assim a condução do impulso que originaria a dor. $\mathrm{Ou}$ seja, essa teoria postula a existência de um portão no corno dorsal da medula espinhal com função de regular a entrada nociceptiva por meio das fibras aferentes de pequeno diâmetro. 
Complementando esses conhecimentos, Sabino et al. $^{38}$ contribuíram afirmando que a teoria de liberação de opióides endógenos, como a endorfina, tem sido usada para explicar a ação desse método em voltagem de baixa frequência. Existem no organismo 3 tipos de receptores opióides, $\mu$, $\delta$ e K, estes se localizam perifericamente na medula espinhal e também nas áreas que estão envolvidas na via descendente da inibição, que se inclui o núcleo magno da rafe localizado na medula rostral ventral $(R V M)$ e a PAG. A TENS de baixa e também de alta frequência influem na antihiperalgesia no SNC mediada pelos receptores $\mu$ e $\delta$ respectivamente. Entende-se então que distintos estimulos de TENS causam a diminuição da dor por meio da ativação de receptores específicos.

\section{- Técnicas de massoterapia}

Ferreira e Lauretti $^{39}$ apostam na massoterapia, que por sua vez consiste em um tratamento alternativo muito comum nos países ocidentais, sendo o seu uso orientado quando ocorre alguma alteração estrutural. Os efeitos desta técnica são localizados e mecânicos, proporcionando relaxamento muscular e também a sedação da dor, além de influenciar na melhoria da circulação sanguínea e drenagem linfática.

Nessa técnica, corroborando com Ferreira e Lauretti $i^{39}$, Gama ${ }^{40}$ afirma que a dor também se reduz pelo aumento dos níveis dopamina e serotonina. Ela também intervém na qualidade do sono e na diminuição de cortisol no organismo, pois melhora a circulação de opióides endógenos nos vasos sanguíneos. No tratamento da DTM são utilizadas dois tipos de massagem, a extra-oral e a intra-oral, sendo a massagem extra-oral mais utilizada.

Outrossim, Gama ${ }^{40}$ explica que a massagem extra-oral é realizada com movimentos de deslizamento e amassamento na cabeça e no pescoço, dando atenção especial aos músculos masseter e temporal. Já na massagem intraoral, aplica-se movimentos de deslizamento superficial e fricção (circular e transversal), estas manobras são feitas principalmente sobre os músculos masseter e pterigóideo medial, usando as pontas dos $1^{\circ} \mathrm{e}$ $2^{\circ}$ dedos.

Capellini et al. ${ }^{41}$ afirmam, por outro lado, que é importante destacar que a melhora clínica com 0 uso da massagem não depende unicamente da redução da tensão muscular, pois diversos fatores também influenciam na melhora, como por exemplo, a motivação e algumas características psicológicas.
CONCLUSÃO

$$
\text { Desta }
$$$$
\text { forma, }
$$

a disfunção

temporomandibular (DTM) está presente em boa parte da população e é uma das causas mais recorrentes da dor orofacial, sobretudo por envolver os músculos da mastigação e a articulação temporomandibular (ATM). Ainda, existem diversas formas de tratamento, sejam elas farmacológicas, tais como métodos medicamentosos, antiinflamatórios esteroidais e não esteroidais (AINES), antidepressivos, relaxantes musculares e anestésicos locais, e também através de métodos não farmacológicos como acupuntura, toxina botulínica, estimulação nervosa elétrica transcutânea e técnicas de massoterapia.

\section{REFERÊNCIAS}

1. Andrade ED, Silva ARS, Bentes APG, Barbosa CMR, Brito FC, Fiol FS, Groppo F et al. Terapêutica medicamentosa em Odontologia. 3 ed. São Paulo: Artes Médicas; 2014.

2. Tosato JP, Caria PHF. Prevalência de dtm em diferentes faixas etárias. RGO. 2006; 54(3):211-24.

3. Carrara SV, Conti PCR, Barbosa JS. Termo do 1ㅇ Consenso em Disfunção Temporomandibular e Dor Orofacial. Dental Press J Orthod. 2010;15(3):114-20.

4. Okeson JP. Tratamento das Desordens Temporomandibulares e Oclusão. 7 ed. Rio de Janeiro: Elsevier; 2013

5. Bouloux GF. Use of opioids in long-term management of temporomandibular joint dysfunction. J Oral Maxillofac Surg. 2011; 69(7):1885-89.

6. Gil AC. Métodos e Técnicas de Pesquisa. 6 ed. São Paulo: Atlas; 2008.

7. Ministério da Saúde. Documento de referência para o Programa Nacional de Segurança do Paciente Programa. Brasília;2014. 42 p.

8. Agência Nacional de Vigilância Sanitária. O que devemos fazer sobre os medicamentos. São Paulo, 2010. 104 p.

9. Guimarães RS, Taveira CC. Fundamentos de Farmacologia. 2 ed. Brasília: NT Editora; 2014.

10. Sharma S, Tabassum F, Khurana S, Kapoor K. Frontline worker perceptions of medication safety in India. Ther Adv Drug Saf. 2016; $7(6): 248-60$.

11. Teixera TCA, Cassiani SHB. Análise de causa raiz de acidentes por quedas e erros de medicação em hospital. Acta Paul Enferm. 2014;27(2):100-7.

12. Instituto Brasileito de Geografia e Estetística (IBGE). Pesquisas de Orçamentos Familiares. 2012. Disponível em:<http://www.ibge.gov.br/ home/estatistica/populacao/condicaodevida/pof /2008_2009_analise_consumo/pofanalise_2008 _2009.pdf>. Acesso em: 12 jun. 2019. 
13. Lynch ME, Watson PN. The pharmacotherapy of chronic pain: a review. Pain Res Manag.2006;11(1):11-38.

14. Hilário MOE, Terreri MT, Len CA. Nonsteroidal anti-inflammatory drugs: cyclooxygenase 2 inhibitors. J Pediatr. 2006;82(5 Suppl):S206-12.

15. Carvalho WA. Antiinflamatórios não esteroides, analgésicos, antipiréticos e drogas utilizadas na tratamento da gota. In: Silva P. Farmacologia. 8. ed. Rio de Janeiro: Guanabara Koogan; 2010. p. 439-466.

16. Batlouni M. Anti-Inflamatórios não esteroides: efeitos cardiovasculares, cérebrovasculares e renais. Arq Bras Cardiol, 2010; 94(4): 556-63.

17. Dym $H$, Israel $H$. Diagnosis and treatment of temporomandibular disorders. Dent Clin North Am. 2012;56(1):149-61.

18. Yagiela JA, Dowd, FJ, Johnson, BS, Mariotti, AJ, Neidle, EA et al. Farmacologia e Terapêutica para Dentistas. 6 ed. Rio de Janeiro: Elsevier; 2011.

19. Katzung BG, Trevor AJ. Farmacologia Básica e Clínica. 12 ed. Porto Alegre: Amgh; 2014.

20. Vicent A, Loffi AOB, Nesi $H$. Uso de corticosteroide no pré-operatório em cirurgia de terceiros molares. Rev bras odontol. 2013;70(1):22-7.

21. Garrote CEM, Teixeira ML, Ramacciato JC, Motta, RHL. Antidepressivos em Odontologia: indicações e cuidados. Rev Assoc Paul Cir Dent. 2010;64(4):294-95.

22. Melo GM. Terapia farmacológica em disfunções temporomandibulares: uma breve revisão. Dentística online. 2011;10(21):36-40.

23. Medawar CV, Matheus ME. Antidepressivos Tricíclicos e Gabapentinóides: uma análise do perfil farmacológico no tratamento da dor neuropática. Rev. Bras. Farm. 2012; 93(3):290-97.

24.Goodman LS, Gilman A. As bases farmacológicas da terapêutica. 11.ed. Porto Alegre: Amgh; 2010. p.460-61.

25. Cascaes LHFS, Oliveira JC. Evidências sobre relaxantes musculares de uso ambulatorial: Uma revisão da literatura. Rev Bras Med Fam Comunidade. 2017;12(39):1-14.

26. Rang HP, Ritter JM. Farmacologia. 8. ed. Rio de Janeiro: Elsevier; 2016.

27. Veering B. Complications and local anaesthetic toxicity in regional anaesthesia. Curr Opin Anaesthesiol. 2003;16(5):455-59

28. Muri EMF, Sposito MMM, Metsavaht L. Efeitos secundários potencialmente desejáveis dos anestésicos locais. Acta Fisiatr.2010; 17(1):28-33.

29.Zotelli VRL, Meirelles MOMR, Souza ML. R. Uso da acupuntura no manejo da dor em pacientes com alterações na articulação temporomandibular (ATM). Rev Odontol Universidade Cidade de São Paulo.
2010;22(2):185-8.

30. Borin GS, Corrêa EC, Silva AMT, Milanesi JM. Acupuntura como recurso terapêutico na dor e na gravidade da desordem temporomandibular. Fisioter Pesq. 2011;18(3):217-22.

31. Florian MR, Meirelles MPMR, Sousa MLR. Disfunção temporomandibular e acupuntura: uma terapia integrativa e complementar. Odontol Clín-Cient. 2011;10(2):189-92.

32. Oliveira CSV. Tratamento de DTM musculares com toxina botulínica-A [monografia]. Curitiba: Universidade Federal do Paraná - UFPR; 2013.

33. Gonçalves BM. Uso da toxina botulínica em Odontologia [monografia]. Florianópolis: Centro de Ciências da Saúde - UFSC;2013.

34. Bicalho MB, Delgado FPE, Borini CB. Toxina botulínica no tratamento da disfunção temporomandibular. Polit Saúde Coletiva. 2015;1(2):153-61.

35. Dall' Antonia M, Oliveira Netto RM, Sanches ML, Guimarães AS. Dor miofascial dos músculos da mastigação e toxina botulínica. Rev dor. 2013;14(1):52-7.

36. Almeida AM. Estimulação Nervosa Eléctrica Transcutânea (TENS) no tratamento da Disfunção Temporo-Mandibular [dissertação]. Lisboa: Universidade de Lisboa;2012.

37. Grossmann E, Tambara JS, Grossmann TK, Siqueira JTT. O uso da estimulação elétrica nervosa transcutânea na disfunção temporomandibular. Rev dor. 2012; 13(3):271-76.

38. Sabino GS, Santos CM, Francischi JN, de Resende MA. Release of endogenous opioids following transcutaneous electric nerve stimulation in an experimental model of acute inflammatory pain. J Pain. 2008;9(2):157-63.

39. Ferreira ASM, Lauretti GR. Massoterapia como técnica no controle da dor em pacientes oncológicos sob cuidados paliativos. Rev Pratica hospitalar. 2007;9(53):161-63.

40.Gama MCS. Efeito de técnicas de massoterapia no tratamento da DTM miogênicas: um estudo clínico randomizado placebo controlado. Piracicaba: Faculdade de Odontologia de Piracicaba - UNICAMP; 2012.

41. Capellini VK, Souza GS, Faria CRS. Massoterapia para o tratamento da DTM miogênica: um estudo piloto. J Appl Oral Sci. 2006;14(1):21-6. 


\section{CONFLITO DE INTERESSES}

Os autores declaram não haver conflitos de interesse

\section{AUTOR PARA CORRESPONDENCIA}

José Henrique de Araújo Cruz

Rua Paulo Diogenes, 57 - Centro

59990000 Rafael Fernandes - RN, Brasil

Telefone: (83) 99625-0125.

E-mail: henrique_araujo1992@hotmail.com

Submetido em 15/10/2019

Aceito em 22/10/2020 